\title{
Volcanic outgassing rates for a stagnant lid Archean Earth: results from a coupled mantle convection, melt partitioning, and volatile speciation model
}

\author{
CLAIRE MARIE GUIMOND ${ }^{1}$, LENA NOACK ${ }^{2}$, GIANLUIGI \\ ORTENZI $^{3}$ AND FRANK SOHL ${ }^{3}$ \\ ${ }^{1}$ University of Cambridge \\ ${ }^{2}$ Freie Universität Berlin \\ ${ }^{3}$ German Aerospace Center, Institute of Planetary Research \\ Presenting Author: lena.noack@fu-berlin.de
}

Understanding how the Archean atmosphere evolved requires constraining mantle outgassing rates, for which observational proxies are rare and not unambiguous, yet for which modelling efforts depend quite strongly on both the presumed tectonic regime and the mantle oxygen fugacity $\left(\mathrm{fO}_{2}\right)$. In particular, switching from a stagnant lid to a mobile lid regime could raise outgassing rates by an order of magnitude. However, Archean climate models will still often adopt outgassing rates in line with modern plate tectonics, despite the contested timeline of plate tectonics' onset. To provide a counterbalance, we have modelled early Earth's C-O-H outgassing if global plate tectonics had not yet initiated and if all outgassing were subaerial, for a wide range of mantle $\mathrm{fO}_{2}$, extrusive-to-intrusive volcanism ratios, and initial conditions. Our numerical model couples 2D compressible mantle convection, redox partitioning in the melt, and volatile chemical speciation. Out of the input parameters studied, mantle $\mathrm{fO}_{2}$ shows the largest effect on the gas fluxes of individual species because it controls both the volatile speciation and (for carbon) the partitioning in the melt. Hence virtually no $\mathrm{CO}_{2}$ would be outgassed below the iron-wüstite mineral redox buffer: in such reducing conditions, (i) carbonate can only partition into the melt in very limited amounts, and (ii) almost all carbon takes the form of $\mathrm{CO}$ instead of $\mathrm{CO}_{2}$. In contrast, the partitioning of water in the melt depends on its mantle source concentration, so the initial water budget has a non-negligible effect on $\mathrm{H}_{2} \mathrm{O}$ and $\mathrm{H}_{2}$ outgassing rates. Overall, our stagnant lid model finds average $\mathrm{CO}_{2}$ outgassing rates of not more than $\sim 1 \mathrm{Tmol} / \mathrm{yr}$, even for oxidized scenarios near the quartz-fayalite-magnetite buffer. This could imply that any reliance on a massive $\mathrm{CO}_{2}$ greenhouse to warm the early Earth might also contain a statement about either the survival of a primary atmosphere or the contemporaneous tectonic regime. 